\title{
Qualidade de vida pós-transplante de medula óssea: comparação entre avaliação das
} crianças e das mães

\section{Quality of life after bone marrow transplant: comparison between evaluation of children and mothers}

\section{Calidad de vida de post-trasplante de médula ósea: comparación entre la evaluación de los niños y las madres}

Recebido: 25/02/2018

Aprovado: 15/08/2018

Publicado: 05/11/2018

\author{
Érika Arantes de Oliveira-Cardoso ${ }^{1}$ \\ Juliana Tomé Garcia ${ }^{2}$ \\ Ana Luisa Carvalho Guimarães ${ }^{3}$ \\ Maria Laura de Paula Lopes Pereira ${ }^{4}$ \\ Jorge Henrique dos Santos ${ }^{5}$ \\ Manoel Antônio dos Santos 6
}

Este estudo teve por objetivo avaliar a qualidade de vida de crianças submetidas ao transplante de medula óssea e comparar os resultados da autoavaliação com o heteroavaliação realizada pelas mães. A amostra foi composta por 14 participantes, sete mães e seus respectivos filhos. Utilizou-se o Questionário Pediátrico de Qualidade de Vida PedsQL $\mathrm{L}^{\mathrm{TM}}$. Os resultados indicam diferença significativa nos valores da avaliação das mães e crianças, sendo que mães superestimaram a QV dos filhos. Este indicador é importante porque sugere necessidade de implementar intervenções com as díades, visando facilitar o diálogo e ampliar a compreensão das vivências emocionais das crianças pelas mães.

Descritores: Qualidade de vida; Criança; Mães; Transplante de Medula Óssea.

This study aimed to evaluate the quality of life of children submitted to bone marrow transplantation and compare the results of self-assessment with the hetero-evaluation carried out by the mothers. The sample was composed by 14 participants, seven mothers and their children. The Pediatric Quality of Life Questionnaire PedsQL $L^{\mathrm{TM}}$. The results indicate significant differences in the values of mothers and children evaluation, and mothers overestimated their children QV. This indicator is important because it suggests the need to implement interventions with the pair, in order to facilitate dialogue and increase understanding of the emotional experiences of children by mothers.

Descriptors: Quality of life; Child; Mothers; Bone marrow transplantation.

Este estudio tuvo como objetivo evaluar la calidad de vida de los niños sometidos a trasplante de médula ósea y comparar los resultados de la autoevaluación con la heteroevaluación realizada por las madres. La muestra consistió de 14 participantes, siete madres y sus respectivos hijos. Se usó el cuestionario de calidad de vida pediátrica de PedsQ $L^{T M}$. Los resultados indican una diferencia significativa en los valores de la evaluación de madres y niños, y las madres sobrestimaron la CV de sus hijos. Este indicador es importante porque sugiere la necesidad de implementar intervenciones con el par, con el objetivo de facilitar el diálogo y ampliar la comprensión de las experiencias emocionales de los niños por las madres. Descritores: Qualidade de vida; Criança; Mães; Transplante de Medula Óssea.

1. Psicóloga. Mestre e Doutora em Psicologia. Orientadora do Programa de Pós-graduação em Psicologia (PPGP) da Faculdade de Filosofia, Ciências e Letras e de Ribeirão Preto da Universidade de São Paulo (FFCLRP-USP), Ribeirão Preto, SP, Brasil. ORCID: 0000-0001-7986-0158 E-mail: erikaao@ffclrp.usp.br

2. Psicóloga. Mestranda pelo PPGP/FFCLRP-USP, Ribeirão Preto, SP, Brasil. ORCID:0000-0003-0265-3331 E-mail: julianatomegarcia@yahoo.com.br

3. Psicóloga. Especialista em Psicologia Hospitalar. Mestre em Psicologia. Psicóloga da Unidade de Terapia Imunológica (Transplante de Medula Óssea) do Hospital das Clínicas da Faculdade de Medicina de Ribeirão Preto (HC-FMRP), SP, Brasil. ORCID: 0000-0001-9967-1856 E-mail: analuisacg@gmail.com

4. Psicóloga. Bióloga. Psicóloga do HC-FMRP, Ribeirão Preto SP, Brasil. ORCID:0000-0002-4482-2482 E-mail: marialaura.pereira@hotmail.com

5. Psicólogo. ORCID:0000-0003-4823-7157 E-mail: jorgecom2r@gmail.com

6. Psicólogo. Mestre e Doutor em Psicologia Clínica. Professor Titular do PPGP / FFCLRP-USP, Ribeirão Preto, SP, Brasil. Bolsista de Produtividade em Pesquisa do CNPq, nível 1B. ORCID:0000-0001-8214-7767 E-mail: masantos@ffclrp.usp.br 


\section{INTRODUÇÃO}

$\mathbf{0}$ Transplante de Medula Óssea (TMO) é um procedimento médico complexo, que tem por princípio básico destruir a medula doente e transferir células progenitoras normais para o indivíduo acometido por neoplasias sólidas $\mathrm{e}$ hematológicas, como leucemias e linfomas, e doenças genéticas e imunológicas. 0 intuito é a reconstituição dos sistemas hematopoiético e imunológico comprometidos, uma vez que o enxerto assumirá a produção das células sanguíneas e contribuirá para a destruição citotóxica das células doentes que ainda permaneceram no organismo do receptor ${ }^{1}$. É indicado para aqueles pacientes que obtiveram pouca resposta ao tratamento biomédico convencional.

Durante o processo de transplante, as crianças enfrentam inúmeros desafios na internação, como a obrigatoriedade de permanecerem em isolamento protetor e de se adaptarem a um esquema rígido de rotinas médicas, além das reações e efeitos colaterais que decorrem da alta toxicidade do tratamento. A expectativa em torno do que pode acontecer, da possibilidade e da ocorrência da dor, das alterações no cotidiano, ocasionam sofrimento por antecipação e, consequentemente, fragilização das crianças e familiares ${ }^{2}$.

Atualmente, o TMO é considerado a terapia mais adequada e bem-sucedida no tratamento de doenças que até há pouco tempo eram fatais, como a leucemia e outras neoplasias hematológicas. No entanto, é um extenso processo, agressivo e prolongado, de grande complexidade e passível de complicações que debilitam, deixam sequelas ou têm consequências fatais. Nesse longo e tortuoso percurso, as crianças confrontam situações e complicações que envolvem dor e sofrimento, tanto de ordem física quanto psicológica².

A radioterapia e a quimioterapia acarretam efeitos colaterais significativos e debilitantes. Complicações decorrentes de quadros infecciosos são possíveis e não muito raras. Componentes do cotidiano das crianças passam a ser controlados por fatores exteriores aos seus desejos. Os atos de comer, dormir e mesmo de se vestir agora se organizam em função de procedimentos médicos e necessidades rigorosas de cuidado ${ }^{2}$.

A criança submetida ao TMO corre o risco de se sentir desapossada de seu corpo e do controle de sua rotina, já que a continuidade de sua vida, de seus vínculos sociais e afetivos foi abruptamente interrompida ${ }^{3}$. Ao mesmo tempo, a família também é acometida por muitos sentimentos negativos, agravados com o estresse produzido pela ruptura da rotina, alterações nas funções e papéis familiares, despesas elevadas e incertezas em relação ao futuro, além das expectativas em relação ao tratamento, seus efeitos adversos e o tempo que é despendido nas idas sucessivas ao hospital ${ }^{4}$.

A criança hospitalizada costuma vivenciar insegurança, desconforto e sofrimento psíquico em função do afastamento dos familiares e de sua casa, dos amigos da escola, da restrição ao leito e do perigo real da morte e é no familiar que ela buscará suporte para enfrentar essa nova realidade 5 .

Logo, perceber-se gravemente doente e necessitar de um tratamento de tamanha complexidade constitui enorme desafio. Elas precisam desenvolver uma nova forma de guiar suas vidas, de adquirir novos conhecimentos e descobrir novas maneiras de ser e de fazer suas existências, enquanto aprendem a conviver com a doença em um ambiente desconhecido e amedrontador.

Os momentos de incertezas que são vividos nessa fase significam que sobreviver à doença e tratamento equivale a viver um dia de cada vez, frente aos desafios e conquistas, às perdas e ganhos diários, em um movimento incessante de buscar novas perspectivas adaptativas. A eficácia do ajustamento à nova realidade depende do apoio familiar e das intervenções recebidas da equipe de saúde, capazes de facilitar o enfrentamento desse período difícil ${ }^{6}$.

Familiares e pacientes têm seu sofrimento intensificado, especialmente pelo fato de que, ao darem entrada à unidade de TMO, já passaram por diversos serviços e se 
submeteram a uma rotina desgastante de exames, tratamentos, consultas e retornos médicos, na busca de dispositivos de saúde que possam oferecer reais possibilidades de cura.

Durante a realização do TMO, a criança enfrenta vários eventos estressores: os efeitos tóxicos das sessões de quimioterapia, as diversas implicações que o tratamento infringe em sua autonomia e a possibilidade de sofrer complicações consequentes ao procedimento. Esses eventos vitais, em conjunto com o risco iminente de perda da vida, podem resultar em prejuízos para a qualidade de vida (QV) de pacientes e familiares ${ }^{1}$.

Indicadores de QV têm sido considerados essenciais para avaliar o impacto da doença e do tratamento na vida do paciente. Também têm sido utilizados para estabelecer indicadores de gravidade $\mathrm{e}$ progressão do agravo, bem como para criar estratégias para minimizar os transtornos acarretados pelo tratamento ${ }^{7}$.

Recentemente, são identificadas duas tendências ${ }^{1-7}:$ QV como um conceito genérico e $Q V$ relacionada à saúde (QVRS). 0 segundo termo é utilizado como um conceito que se refere especificamente a disfunções ou agravos à saúde, incluindo a avaliação de aspectos diretamente relacionados às doenças e às intervenções em saúde, como limitações ou incapacidades e seus efeitos na percepção do bem-estar7.

Avaliar a QVRS das crianças póstratamento é crucial para subsidiar ações em saúde. Nos últimos anos, há uma preocupação em analisar a concordância da QVRS aferida pela criança e a avaliada pelos pais. A importância desse tipo de mensuração é a de promover ações que realmente sejam mais efetivas para a saúde, física e mental das crianças, segundo as suas próprias necessidades, não as percebidas ou vivenciadas pelos pais, que acabam sendo os "porta-vozes" dos filhos. Apesar de não haver um resultado convergente, em todos os estudos aparecem divergências entre a auto e a heteroavaliação $0^{8-16}$.

Os resultados da QVRS, tanto auto como heteroavaliados, são muito diferentes, dependendo do contexto no qual foi realizado o estudo. Foram encontrados em língua portuguesa dois artigos sobre o tema, um de validação do instrumento no cenário brasileiro $^{9}$ e outro no contexto português, sendo este um estudo descritivo realizado com crianças com câncer com idades entre oito e 17 anos; verificou-se diferença significante entre a percepção da criança sobre sua QV $(66,0 \pm 13,3)$ e a de seus pais $(60,3 \pm 15,0)^{17}$.

Este estudo teve por objetivo avaliar a qualidade de vida de crianças submetidas ao transplante de medula óssea e comparar os resultados da autoavaliação com o heteroavaliação realizada pelas mães.

\section{MÉTODO}

Estudo descritivo e exploratório, de corte transversal. Foram incluídas todas as crianças que realizaram o TMO e se encontravam na fase pós-alta hospitalar (até uma semana da saída da enfermaria), no período de janeiro a dezembro de 2015, além de suas respectivas mães.

Os participantes estavam em acompanhamento ambulatorial na Unidade de TMO de um hospital terciário do interior do Estado de São Paulo.

Os critérios de inclusão foram: criança recém-submetida ao transplante, ter entre oito e 11 anos, estar no período de alta hospitalar com acompanhamento ambulatorial e apresentar, juntamente com sua mãe, disponibilidade para colaborar de maneira voluntária com a pesquisa. Foram avaliadas todas as crianças que tiveram alta da enfermaria da Unidade de TMO.

0 instrumento utilizado foi o Pediatric Quality of Life Cancer Module (PedsQL ${ }^{\mathrm{TM}}$ ). Trata-se de um instrumento validado para o Brasil para avaliar crianças e adolescentes com câncer, capaz de aferir como a enfermidade e o método terapêutico adotado interferem na QVRS das crianças ${ }^{18}$.

Estruturalmente, é composto por 27 itens, distribuídos em oito subescalas: dor e ferimentos (dois itens), náusea (cinco), ansiedade frente ao procedimento (três), ansiedade frente ao tratamento (três), preocupações (três), dificuldades cognitivas 
(cinco), percepção da aparência física (três) e comunicação (três). A escala do tipo Likert é constituída por cinco opções de resposta: "nunca", "quase nunca", "algumas vezes", "muitas vezes", "quase sempre"18.

0 PedsQL ${ }^{\mathrm{TM}}$ foi aplicado individualmente. 0 pesquisador leu as questões para a criança, na íntegra $\mathrm{e}$ literalmente (por exemplo: Eu sinto dor, eu me machuco nas minhas "juntas" [articulações] $e$ músculos). As mães também completaram o instrumento, composto das mesmas questões, porém fazendo referências a seus filhos (por exemplo: Meu filho/Minha filha tem dor ou machucado nas "juntas" [articulações] $e$ músculos).

A coleta de dados foi realizada em ambiente preservado da instituição hospitalar, sempre que possível em sala reservada, resguardando-se os princípios de conforto e privacidade, com duração de, aproximadamente, 20 minutos.

Os dados foram analisados de acordo com as recomendações do instrumento. Após a aplicação atribuiu-se um escore para cada questão que, posteriormente, foi transformado em uma escala de 0-100, em que o zero corresponde a um pior estado de saúde e 100 a um melhor, sendo cada dimensão analisada separadamente. Os valores obtidos pelas crianças foram comparados com os das mães pela utilização do teste Mann-Whitney. 0 nível de significância adotado foi $\mathrm{p} \leq 0,05$.

$\mathrm{O}$ projeto foi aprovado pelo Comitê de Ética em Pesquisa (CAEE no 23198513.3.0000.5407).

\section{RESULTADOS}

A amostra foi composta por 14 participantes, sendo sete crianças e suas respectivas mães. Observa-se pouca variação na idade dos participantes, sendo o diferenciador maior o diagnóstico. Todas as mães exerceram a função de cuidadoras, em tempo integral, dos filhos durante o Transplante de Medula Óssea.

Tabela 1. Caracterização de crianças pós TMO. Ribeirão Preto, SP, Brasil, 2016.

\begin{tabular}{lcll}
\hline Sexo & Idade* $^{*}$ & Diagnóstico & Idade da mãe \\
\hline $\mathrm{F}$ & 10 & Anemia Falciforme & 31 \\
$\mathrm{~F}$ & 10 & Anemia Aplástica Grave & 33 \\
$\mathrm{M}$ & 8 & Leucemia Linfóide Aguda & 39 \\
$\mathrm{M}$ & 10 & Anemia Aplástica Grave & 32 \\
$\mathrm{M}$ & 9 & Anemia Falciforme & 38 \\
$\mathrm{M}$ & 10 & Anemia Aplástica Grave & 32 \\
$\mathrm{~F}$ & 10 & Anemia Falciforme & 33 \\
\hline * Idade em anos na data da coleta &
\end{tabular}

Os resultados obtidos na avaliação dos diferentes domínios da QVRS pelo PedsQL ${ }^{\mathrm{TM}}$, na perspectiva das crianças e das mães, foram sistematizados na Tabela 2, que permite uma comparação entre a auto e heteroavaliação.

Tabela 2. Comparação entre a auto e a heteroavaliação da QVRS das crianças submetidas ao TMO. Ribeirão Preto, SP, Brasil, 2016.

\begin{tabular}{lccccc}
\hline \multicolumn{1}{c}{ Domínio } & \multicolumn{2}{c}{ Filhos } & \multicolumn{3}{c}{ Mães } \\
\hline Dores & M & DP & M & DP & $P$ \\
Náuseas & 70,4 & 22,1 & 97,9 & 4,6 & $0,03^{*}$ \\
Ansiedade procedimento & 70,8 & 22,6 & 79,1 & 21,8 & 0,42 \\
Ansiedade tratamento & 72,0 & 23,3 & 48,5 & 32,0 & 0,49 \\
Preocupações & 76,0 & 28,5 & 84,6 & 15,4 & 0,95 \\
Funções cognitivas & 64,3 & 30,7 & 82,0 & 11,5 & 0,63 \\
Aparência & 66,6 & 30,7 & 70,8 & 28,1 & 0,81 \\
Comunicação & 66,6 & 28,3 & 84,7 & 13,1 & 0,56 \\
Total & 68,0 & 45,2 & 67,9 & 24,2 & 0,64 \\
M = média, DP = desvio-padrão & * Diferença estatisticamente significativa & & & & $\mathbf{0 , 2 6}$ \\
\hline
\end{tabular}


De modo geral, a autoavaliação da criança $(69,3 \pm 13,4)$ sobre sua QVRS foi pior do que a estimada pelas mães $(76,9 \pm 13,7)$. Observou-se diferença estatisticamente significativa nos valores da avaliação das mães e das crianças no componente Dores ( $p$ $=0,03$ ), sendo que as mães superestimaram a ausência de dor nos filhos.

Os domínios da PedsQL ${ }^{\mathrm{TM}}$, na perspectiva das crianças, foram classificados da seguinte forma, em ordem decrescente de preservação: (1) Ansiedade com tratamento, (2) Ansiedade com procedimento, (3) Náuseas, (4) [ausência de] Dores, (5) Comunicação, (6) Funções cognitivas, (7) Aparência, (8) Preocupações. Na perspectiva das mães: (1) [ausência de] Dores, (2) Aparência, (3) Ansiedade com tratamento, (4) Preocupações, (5) Náuseas, (6) Funções cognitivas, (7) Comunicação e (8) Ansiedade com procedimento.

Analisando o domínio Dores da PedsQL ${ }^{\mathrm{TM}}$, chama a atenção a diferença estatisticamente significativa $(p \leq 0,05)$ entre a frequência com que a criança relata sentir dores $(70,4 \pm 22,1)$ e a frequência com que a mãe acredita que o filho vivencia estímulos dolorosos $(97,9 \pm 4,6)$.

A totalidade das mães endossou itens que indicam que o filho nunca sente dores nas articulações e músculos, enquanto que as respostas dos filhos se dividem nas demais gradações, sendo que três deles afirmaram sentir dores "muitas vezes" e "quase sempre".

o único domínio no qual as mães subestimam a respostas dos filhos é o da Ansiedade frente aos procedimentos, que se refere às dificuldades de se submeterem a intervenções que envolvem agulhas, injeções e exames. Destaca-se o fato de que foi o domínio que obteve menor média na estimativa das mães $(48,5 \pm 32,0)$ e o segundo melhor avaliado pelas crianças $(72,0 \pm 23,3)$. As respostas das crianças se concentram em "nunca" e "quase nunca" sentem dificuldades com esses procedimentos. Já as mães acreditam que os filhos ficam ansiosos "muitas vezes" e "quase sempre" diante desses procedimentos invasivos.

O componente da QVRS no qual os dois grupos mais se aproximaram foi Comunicação. Este domínio da PedsQL ${ }^{\mathrm{TM}}$ é compreendido como a facilidade em conversar - com a equipe de saúde e com pessoas de um modo geral - sobre a doença e a respeito do que sentem em relação à sua condição e ao tratamento. No entanto, no ranking dos domínios, Comunicação ficou em quinto lugar na percepção das mães e em penúltimo (sétimo) lugar na autoavaliação dos filhos, sugerindo que há dificuldades no estabelecimento de diálogo sobre essas questões cruciais para o tratamento.

\section{DISCUSSÃO}

Os resultados mostram que a autoavaliação da criança sobre sua QVRS foi pior do que a estimada pelas mães. Esses dados corroboram a outras pesquisas, que além de mostrar divergências entre as duas pontuações, sinaliza uma tendência de melhor avaliação pelos pais do que pelas próprias crianças, isto como uma tendência dos pais em projetarem nos filhos a sua própria QVRS ${ }^{8,12,14}$.

No presente estudo houve baixa pontuação, em especial as obtidas pelas crianças, quando comparadas às referidas nos contextos de outros países, como um estudo norte-americano que relatou um escore médio de autoavaliação de 73 e de heteroavaliação $74^{8}$. No contexto japonês as médias das crianças foram 78 e da família $75^{14}$ e na China as crianças pontuaram 84 e os pais $80^{12}$.

Os dados do presente estudo se aproximam mais dos resultados encontrados no cenário português, em termos da média da QVRS percebida pela criança $(66,0 \pm 13,3)$, não obstante se distanciem no que tange à avaliação dos pais $(60,3 \pm 15,0)^{17}$. Ou seja, os pais portugueses reportam uma QVRS de seus filhos com câncer, bem mais depreciada do que as mães brasileiras.

Os estudos mencionados não se referem à realidade do TMO, procedimento que reconhecidamente implica em prejuízos na QV do paciente e do familiar imediatamente após sua realização ${ }^{1-19}$. Esse comprometimento é de tal magnitude que os autores desenvolveram um módulo da PedsQL ${ }^{\mathrm{TM}}$ específico para TMO, levando em consideração as implicações e complicações do próprio procedimento ${ }^{20}$. Essa escala ainda 
não está disponível para uso na língua portuguesa.

No presente estudo, destaca-se como mais preservado o domínio Ansiedade frente ao tratamento. Esse resultado foi também encontrado em outros estudos, resultados estes relacionados à esperança na resolutividade do procedimento ${ }^{8-17}$. Entre os domínios menos preservados aparece a insatisfação com a aparência física, relacionada às alterações da imagem corporal em decorrência do tratamento, que nem sempre são consideradas para a realidade infantil. Essas alterações muitas vezes são colocadas como um problema exclusivo do universo juvenil e adulto, inclusive pelas mães que atribuíram elevada pontuação para esse componente $(84,7 \pm 13,1)$, quando comparada às respostas das crianças $(66,6 \pm 28,3)$.

Três domínios sobressaíram-se nos resultados: Dor (mães subestimam as dores vivenciadas pelos filhos), Ansiedade frente aos procedimentos (mães superestimam as dificuldades dos filhos frente à necessidade de exames) e Comunicação (domínio no qual se obteve maior aproximação entre as respostas dos dois grupos). A tendência a perceber como menor a intensidade da dor vivenciada pelos filhos foi encontrada em outro estudo ${ }^{21}$, que investigou 251 crianças (92 com câncer) e seus pais, com o mesmo instrumento utilizado no presente estudo.

A avaliação da dor é subjetiva e pode afetar diferentes esferas da QV do paciente, e que a mãe, em especial, é peça importante no manejo desse sintoma, sendo, portanto, de fundamental importância que ela compreenda a real dimensão com que a criança vivencia sua experiência álgica ${ }^{21}$.

0 profissional de saúde necessita estar atento ao envolvimento de aspectos psicológicos aliados aos processos fisiológicos na manifestação da dor. A assistência deve ser baseada na avaliação da história clínica informada pelo próprio paciente, características de personalidade, crenças sobre dor, limitações funcionais e desenvolvimento de estratégias para o enfrentamento da experiência dolorosa. Sabese que fatores psicológicos têm uma poderosa influência no tratamento de pacientes com dor crônica.

Quanto às dificuldades em relação aos procedimentos considerados como invasivos (domínio Ansiedade frente ao procedimento), estudo mostrou que a sensação de falta de controle, sentida pelos pais, sobre as intervenções dolorosas afeta sua QV e pode interferir na percepção da vivência desse domínio pelos filhos ${ }^{21}$.

Em relação à Comunicação, pode-se interpretar como positivo o resultado obtido, uma vez que tanto os filhos como as mães mostraram uma percepção semelhante em relação às dificuldades e facilidades desse processo, que é de importância quando se pensa na possibilidade de se implementarem intervenções em relação aos demais domínios discrepantes.

\section{CONCLUSÃO}

A avaliação da QVRS é considerada importante para a condução e humanização do tratamento oncológico. Os resultados da PedsQL ${ }^{\mathrm{TM}}$ mostram que há um descompasso entre a percepção da QVRS das crianças e de suas mães.

A equipe necessita estar especialmente atenta à percepção materna da ausência dor na criança, em comparação com a avaliação da vivência em relação aos procedimentos dolorosos do pós-TMO na perspectiva dos próprios filhos.

0 fato de as mães superestimarem a ausência de dor de seus filhos pode levar a comportamentos agressivos por parte destes, aparentemente de difícil compreensão, em especial em relação aos profissionais que executam os procedimentos invasivos e dolorosos.

A presente pesquisa apresenta limitações, como amostra reduzida e delineamento transversal; no entanto, como primeiro estudo exploratório no contexto brasileiro com crianças do TMO, oferece resultados relevantes para futuras investigações com amostras mais robustas e desenho longitudinal.

Quanto à presença e intensidade da dor, faz-se necessária uma avaliação conjunta da dupla mãe-filho, com uso de instrumentos 
específicos, de modo a se obter um dimensionamento mais preciso deste constructo tão subjetivo.

Os resultados sugerem que a equipe de saúde necessita implementar intervenções que favoreçam a aproximação e o diálogo entre mães e crianças, de modo que estas possam ser sensibilizadas para conhecer as reais necessidades vivenciadas pelos filhos.

Dessa maneira, os profissionais poderão ajudar as mães a atuarem de forma mais efetiva na manutenção da QVRS das crianças e a sua própria, uma vez que ambas são interrelacionadas.

\section{REFERÊNCIAS}

1. Crovador LF, Cardoso EAO, Mastropietro AP, Santos MA. Qualidade de vida relacionada à saúde de pacientes com esclerose múltipla antes do TCTH. Psicol Reflex Crític. 2013; 26(1):58-67.

2. Mazza VA, Souza C, Estevão AR, Guimarães SL, Mercê NNA. Vivência de famílias de crianças e adolescentes submetidos ao transplante de células-tronco hematopoéticas. Rev Eletrônica Enferm. [Internet]. 2016 [citado em: 15 jul 2017]; 18:1-11.

$$
\text { Disponível }
$$

em:

http://doi.org/10.5216/ree.v18.40131

3. Freitas IS, Oliveira-Cardoso EA, Santos MA. Espiritualidade e religiosidade em mães de crianças com câncer hematológico. Psicol Estud. 2017; 22(1):433-47.

4. Oliveira-Cardoso EA, Santos MA. Luto antecipatório em pacientes com indicação para o Transplante de Células-Tronco Hematopoéticas. Ciênc Saúde Colet. 2013; 18(1):2567-75.

5. Ribeiro JP, Gomes GC, Thoferhrn MB, Mota MS, Cardoso LS, Cecagno S. Criança hospitalizada: perpectivas para o cuidado compartilhado entre enfermagem e família. RevEnferm UFSM. 2017; $7(3): 350-62$.

6. Anders JC, Rosa LM, Souza AIJ. Cuidados de enfermagem à criança e ao adolescente submetidos a transplante de células-tronco hematopoéticas. In: Associação Brasileira de Enfermagem; Gaíva MAM, Ribeiro CA, Rodrigues EC, organizadores. PROENF- Programa de atualização em Enfermagem: saúde da criança e do adolescente. 2015; 2(1):111-153. (Sistema de Educação Continuada à Distância, v. 2).

7. Seidl EMF, Zannon CMLC. Qualidade de vida e saúde: aspectos conceituais e metodológicos. Cad Saúde Pública. 2004; 20(2):580-8.

8. Varni JW, Burwinkle TM, Katz ER, Meeske K, Dickinson P. The PedsQl in pediatric cancer: reliability and validity of the Pediatric Quality of
Life Inventory Generic Core Scales Multidimensional Fatigue Scale and Cancer Module. Cancer. 2002; 94(1): 2090-106.

9. Felder-Puig R, Frey E, Proksch K, Varni JW, Gadner H, Topf R, et al. Validation of the German version of the Pediatric Quality of Life Inventory (PedsQL) in childhood cancer patients off treatment and children with epilepsy. Qual Life Res. 2004; 13(1):223-34.

10. Scarpelli AC, Paiva SM, Pordeus IA, RamosJorge ML, Varni JW, Allison PJ. Measurement properties of the Brazilian version of the Pediatric Quality of Life Inventory [PedsQL (TM)] cancer module scale. Health Qual Life Outcomes. 2008; 6:7.

11. Sitaresmi MN, Mostert S, Gundy CM, Sutaryo, Veerman AJP. Health-related quality of life assessment in Indonesian childhood acute lymphoblastic leukemia. Health Qual Life Outcomes. 2008; 6:96.

12. Lau JTF, Yu X -n, Chu Y, Shing MMK, Wong EMC, Leung TF, et al. Validation of the chinese version of the Pediatric Quality of Life Inventory TM (PedsQLTM) Cancer Module. J Pediatr Psychol. 2009; 35(1):99-109.

13. Ji Y, Chen S, Li K, Xiao N, Yang X, Zheng S, et al. Measuring health-related quality of life in children with cancer living in Mainland China: feasibility, reliability and validity of the chinese Mandarin version of PedsQL 4.0 Generic Core Scales and 3.0 Cancer Module. Health Qual Life Outcomes. 2011; 23(9):103-16.

14. Tsuji N, Kakee N, Ishida Y, Asami K, Tabuchi K, Nakadate $\mathrm{H}$, et al. Validation of the Japanese version of the Pediatric Quality of Life Inventory (PedsQL) Cancer Module. Health Qual Life Outcomes. 2011; 9(22):1-16.

15. Chaudhry Z, Siddiqui S. Health related quality of life assessment in Pakistani pediatric cancer patients using PedsQL ${ }^{\mathrm{TM}} 4.0$ generic core scale and PedsQL ${ }^{T M}$ cancer module. Health Qual Life Outcomes. 2012; 10:52.

16. Kuhlthau KA, Pulsifer MB, Yeap BY, Rivera Morales D, Delahaye J, Hill KS, et al. Prospective Study of Health-Related Quality of Life for Children With Brain Tumors Treated With Proton Radiotherapy. J ClinOncol. 2012; 30(17):2079-86. 17. Batalha LMC, Fernandes AM, Campos C. Quality of life among children with cancer: agreement between child and parent reports. Esc Anna Nery Rev Enferm. 2015; 19(2):292-6.

18. Botelho ACSR. Validação para o uso no Brasil do "Pediatric Quality of Life ${ }^{\text {TM" }}$ (PedsQLTM): um estudo envolvendo famílias com crianças e adolescentes acometidos por câncer. [dissertação]. Belo Horizonte: Faculdade de 
Odontologia, Universidade Federal de Minas Gerais; 2007.

19. Prado FAC, Cardoso EAO, Zuben BVV, Santos MA. Oficinas de atividades: reconstruindo o cotidiano de pacientes submetidos ao TMO. Rev Cult Ext. 2015; 12(1):86-96.

20. Lawitschka A, Guclu ED, Varni JW, Putz M, Wolff D, Pavletic S, et al. Healty-related quality of life in pediatric patients after allogenic SCT: development of the PedsQL Stem Cell Transplant module and results of a piloty study. Bone Marrow Transplant. 2014; 49(1):1093-7.

21. Matziou V, Vlachioiti E, Megapanou E, Ntoumou A, Dionisakopouloi C, Dimitriou V. Perceptions of children and their parents about the pain experienced during their hospitalization and its impact on parent's quality of life. Jpn J Clin Oncol. 2016; 46(5):453-61.

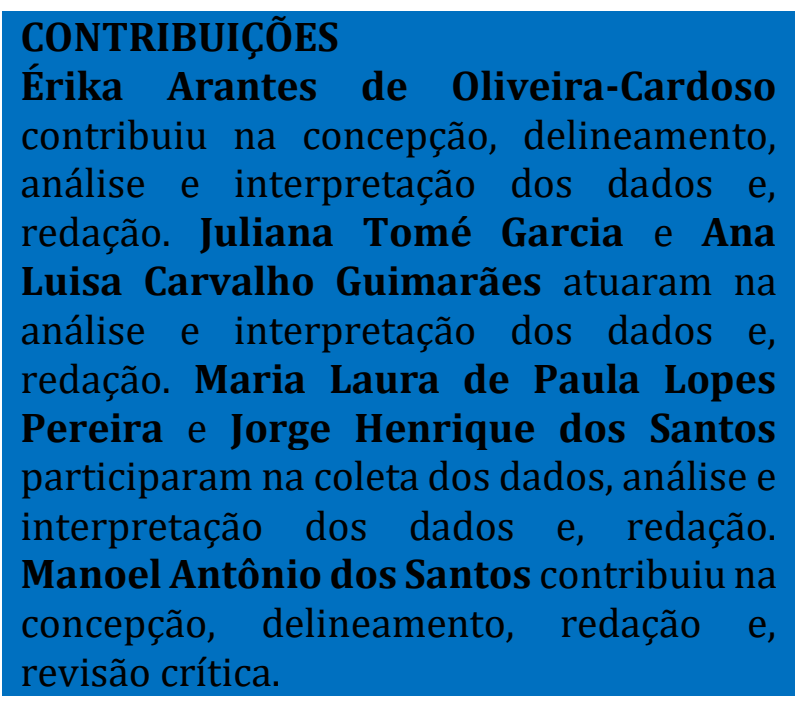

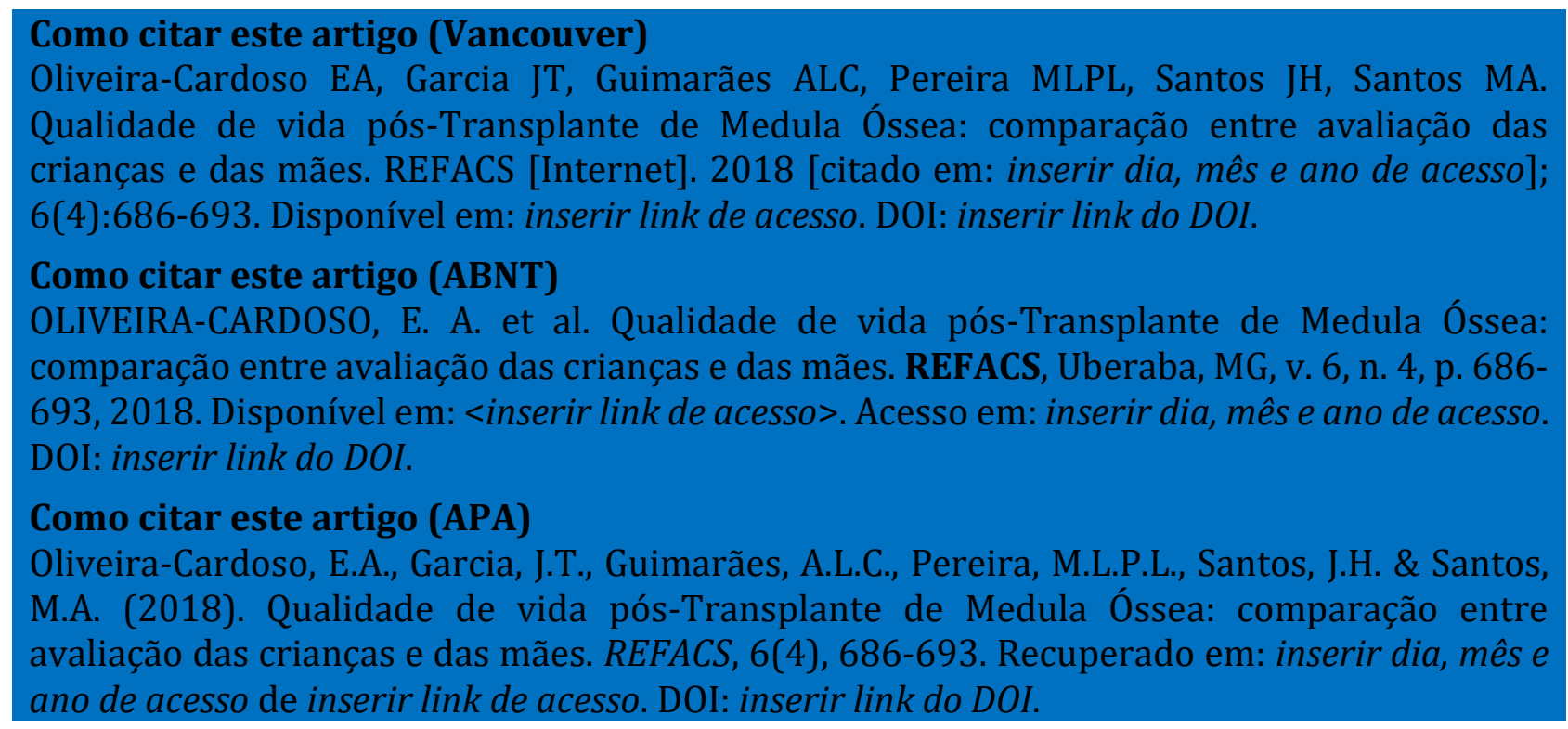

